les concessionnaires de celle entreprise est bienveillante. Les redevances qu'il impose sont conçues daus un esprit de la plus parfaite appréciation des devoirs de l'Etat cnvers une industric naissante.

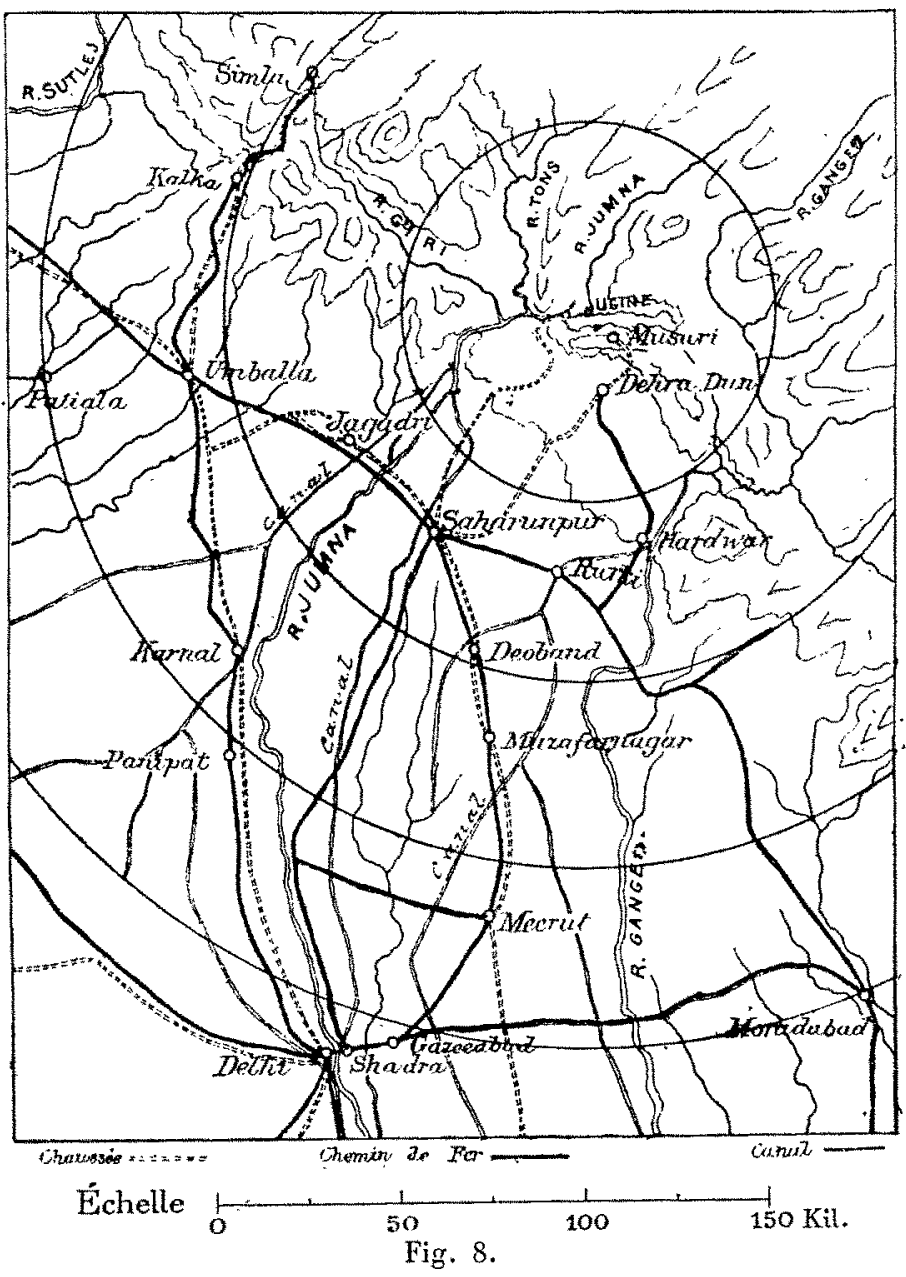

Distributions de Bombay et de Calcutta

Tous devons mentionner ici l'entreprise de la maison Tara, de Bombay, qui se propose de fournir l'énergie ćlectrique à la grande ville de Bombay, tant pour la force notrice nécessaire aux fabriques el la traclion, que pour assurer l'ćclairage électrique. C'est un projet hydro-électrique avec emploi de réservoirs pour alimenter une chule de $520 \mathrm{~m}$. de hauteur, capable de produire 50 ooo IIP, qui doivent être livrés, après les pertes inćvilables, à une distance de $68 \mathrm{~km}$. C'est une entreprise importante qui doil être commencée sois peu.

Signalons enfin un projel qui est à l'étude pour cxploiter une chute d'eau, au Molnbavci, et transporter l'énergie produite à Calculla distant de 2 fo kilomètres.

Pour terminer, nous vouhrions dire un mot sur l'allitude dans ce pays des fournisseurs de malériel et d'appareillage ćlechiques. Leur développement pendant ces vingt dernières années est remarquable. On a vu un marchand de verrerie et de lustres transformé en fournisseur d'appareils électriques, ainsi qu'une simple maison de commerce s'affubler du ròle d'ingénieur électricien. Cela vient sans dou!e avec le mouvement commercial, de même que l'on voit, à Paris, un fameux carrossier devenir renommé pour ses automobiles. Cela dépend beaucoup comme l'on se trouve outillé pour entreprendre un nouveau mélier.

Naturellement, une bonne partie du matériel et de lappareillage importés aur Indes sont de provenance anglaise $\mathrm{d}$, si l'on peut se fier aux chiffres donnés par une certaine revue, il paraît que, en 1go6-1907, la valeur des fournitures et appareils électriques importés était de i 250 ooo franes, et pour l'amnée suivante, le même chiffe global fut maintemu. Les importations de provenance américaine entreraient dans ces évaluations pour un quinzième. Nous avouons resLer sceptique à propos de ces résullals, car celte Revue ne dit rien des importations considérables de provenance allemande ou suisse. Serait-il permis de supposer que leurs montants aient glissé dans le gros tolal athibué aux imporlations anglaises?

Il $y$ a maintenant des maisons anglaises, allemandes, américaines el suisses, élablies aux Indes, principalement ì Bombay et à Calculta, qui s'occupent des fournitures el appareils ćlectriques. Il est regrellable qu'aucun représentint des maisons francaises, qui ont pour spécialité les installations hydro-électriques, ne se soit cncore, que nous sachions, ćlabli dans ce pays. II nous semble, pourtant, que ce soml justement les maisons du pays de la houille blanche, qui s'occupent d'installations hydro-électriques dans un pays difficile, où le transport de pièces lourdes doil être effectué par des chemins de muletiers, comme c'est le cas pour la rógion des Alpes, il nous semble que se sont spécialement de lelles maisons, qui, par leur expérience, seraient les plus capables de fournir le matériel el les fournitures les plus modernes et les plus convenables à de telles entreprises

Beresfond-Lovetr,

Nembre de $l$ " Instilution of Electrical Enginers".

\section{AMÉNAGEMENT DES CHUTES}

\section{UTILISATION DES FORCES MOTRICES DU LAC WALOHEN}

Les lecteur's de La Houille Blanche seront peut-être intér ressés par quelques reiseignements sur les résulals du concours international inslitué par l'Etat bavarois, en vue d'oblenir la meilleure solution possible du problème de l'installation des forces molrices du lac de Walchen $\left(^{*}\right)$.

le lac de Walchen, l'un des plus piltoresques des Mpes bavaroises, est, sur sa rive gauche, séparé par le Kesselberg. du lac de Kochel dont le niveau est à $202 \mathrm{~m}$. plus bas. De rive à rive il n'y a que $2 \mathrm{~km}$, et le tunnel qui permetta de déverser les caux de l'un dans l'autre aura à peine plus de i $\mathrm{km}$. de long. Sur sa rive droile, le Walchensee est séparé de la vallée an fond de laquelle coule l'Isar par le massif montagneux de l'Isarberg dont la base n'a que $3,5 \mathrm{~km}$. do largeur

Rien donc do plus simple que de percer le Kesserberg par un tumnel, el d'utiliser l'cau du Walchensee ef la chule cnlre les deux lacs. Le cours d'eau de la Jachen qui alimente le Walchensce est peu important, el le volume d'eau ainsi utilisable modeste. Mais l'Isar, dans le voisinage du Walchensee. esl déjà un cours d'eau imporlant, el possèle un niveau suf́fisamment élevé pour qu'on puisse, on le détournant, l'amener dans le lac. Il en est de même pour le Rissbach alluent important de l'Isar.

On voit que le problème posé est comparable à celui qui a élé xésolu pour le lac de Joux, mais qu'il cst plus complexe, cl que les résullats à obtenir sonl beaucoup plus importants.

(*) N'après un artic'e de M. J. Micuaud, paru dans le Bulletin technque de la Suisse romande. 
Au Walchensed, il singil d'un débil permanent de 25 à $90 \mathrm{~m}^{3}$ par seconde, sous unc chule brute de $202 \mathrm{~m}$, avec un hac regulateur de $16 \mathrm{~km} .^{2}$ de superficio, landis quau lac de Joux, il n'y a que $1,6 \mathrm{~m}^{3}$ de débit permanent, une chrice de , $40 \mathrm{~m}$., el un lac de $9 \mathrm{~km}^{2}$ de surface. I a dénivellation permise au lac do Joux entre les basses el les haules eaux est de $3^{m 5}$, landis que, au Walchensee, on en prévoil une de $I^{3}$ ì $20 \mathrm{~m}$. suivant les projets. Aussi, tandis qu'au lac de Joux la force permanente est de 3750 chevaux, elle en atloint 50 ono au Walchensee.

Comme loujouns, c'est l'initiative privée qui a atlaché l' grelot. T.e conseiller Schmick, de Darmstadt, et M. Jeanjaquel, ingénieur suisse, furent les premiers à demander uno concession en 100 /. Leur exemple ful suivi à la fin de la même anné par le major Donat. Mais l'Etat bavarois s'empara de l'idée, et fit étudier, par son personnel technique, un projel d'ullisalion de ces forces, en vue surtoul de réalisen progressirement la traclion électrique des chemins de fei de ITtat.

Dans lous ces projets, le lac de Walchen fonctionn: comme accumulaleur. Dans celui du major Donat, on conctitue, a laide de grands barrages, des lacs artificiels su l'lsa: ou ses aflluents. Ces lacs suppléent ou même rempla. cont lo lac de Walchen, comme accumulateur. En ontre, ils creent des chules el des forces supplémentaires. Dans sor ivant-projet, l'administration bavaroise avait dû renoncer : créer des lacs artificiels à cause de la grande difficulté, pour no pas dire l'impossibilité, de construire des barages frò devés el solides dans les terrains d'alluvion sur lesquelu coule l'Isar.

Ces trois projets primitifs contiennent d'ailleurs, en germe, loutes les solutions que le concours international a mises au jour. Le concours a ćté fort brillant. Des articles spéciaux parus dans la Deulsche Bauzeilang, de Berlin, d dans la Schweizerisehe Bauzeitung, de Zurich, en rendert comple.

Trente ot un projets ont été présentés, et six recompensćs. Ce ne sont généralement pas des ceuvres individuelles, mais plutôt des ceuvres de collectivités : ingénieurs civils. archilectes, b́lectriciens el fabriques de machines associés. On peul se faire une idée de la masse énorme de travail effeclui par les concurrents, en rappelant que les $3 \mathrm{r}$ projets comportent ensemble 1416 plans el $x$ i6 mémoires justificatifs. T'écrivain de la Bauzeilung berlinoise estime à 25 oon marks la dépense moyenne faile pour f́laborer chacun des 3 r projels, soit un total de $77^{5}$ ooo marks ; tandis que la rémunération qui leur a été allouée, sous forme de six primes, ne s'élèvo qu'à un total de 60 ooo marks, soit moins du douzième de la dépense. L'évaluation du journal allemand est peut-êtro un peu exagérée ; mais la grandeur colossale du travail présenté subsiste.

Comme le problème à résoudre ne souffrait pas de grandes difficultés purcment lechniques, et que l'altention des concurrents avait été appelée sur le cốó artistique de l'entre. prise, les plans présentés soni extrêmement soignés dans la forme, et chacun des auleurs s'est beaucoup préoccupé de l'eflet architectural des constructions qu'i] proposait.

Estimant suns doute que les étapes successives à parcourir, pour arriver il l'utilisation complète des forces du Walchensee, ne se succéderaient que très à la longue, comme il convient du reste à l'œuvre d'une administration publicjuc, lo programme du concours limitait à $3^{m} 50$ sculement l'abaissement autorisé des eaux du lac pendant la première périodr. quilte à angmenter plus fard col abaissment. Plusieurs projels supposent la création de quais str centaines parlies des rives, avec murs de 4 ou $5 \mathrm{~m}$. de hauleur. Fn admeltant; contrairement aux idées des disciples de Ruskin, que la beaulé du paysage serait mieux conservée de cetle laçón, pendant la première période des travaux toul au moins, on peut se demander si ce ne sera pas plntôt une cause d'enlaidissement pendant les périodes subséquentes où les variations de niveau alteindront $x 3$ on même $20 \mathrm{~m}$., el laisseron! les murs de quai terriblement en l'air.

A côté des éloges mérilés par les concurrents du Walchensee, et relevés à jusle litre dans les deux jommaux cilés plus haut,il naus sera peut-être permis de relever deux points qui nous paraissent critiquables, tout en ronvoyant aux articles des deux Bauzeitungen les lecleurs qui voudraient se renseigner avec quelques délails sur les éléments constitutifs des solutions proposécs, barrages, souterrains, turbines, dynamos, etc.

La première crilique sapplique à lapprécialion de la quanlité d'eau que peurent réellement foumir en temps de basses eaux les trots cours d'cau qui alimenten le Walchensce, et la seconde aux prises d'eau dans leur rapport avec la question de l'ensablement des canaux.

D'après lfs données fournies aux concurrents par l'administration bavaroise, dans les documents du concours, il est admis que le bassin de I'Isar fournit un débit minimum de 7,6 litres par seconde et par $\mathrm{km}^{2}$, celui du Rissbach un débit de 9, r litues, et enfin qu'à la sortie du lac de Walchen le débit peut descendre à 6,8 litres par $\mathrm{km}^{2}$. On remarquem que ces chiffres sont bien plus forts que ceux que l'on a controlés en Suisse, soil f i 5 litres seconde par $\mathrm{km}^{2}$. Collo différence est évidemment possible, sinon vraisemblable.

II faut cependant relever le fait curicux que c'est à la sortie du lac que ce débit minimum par $\mathrm{km}^{2}$ est le moindre. Normalement, ce devrait etre le contraire. En effet, si en hautes eaux et période de crue, le lac peul recevoir justu't $7^{3} \mathrm{~m}^{3}$ par seconde, landis qu'il nen sort que $19 \mathrm{~m}^{3}$, il devrait s'en échapper en basses caux el période de décrue plus d'eau qu'il n'en entre, el par conséquent, selon toule vaisemblance, plus qu'il n'en passe dans les cours d'eau voisins qui nont pas de lac régulaleur. Peul-être y a-t-il, comme au lnc de Joux, des cxutoires invisibles, en sorle que l'affuent visible de Jachen n'emmène pas ì lui seul tout" l'eau qui sort du lac. Cela n'est cependant pas probable.

Pour corroborer ces craintes sur la valeur réelle des débils minima avec lesquels il fant compler, on peut faire la remarque suivante. Le 25 septembre 1006 , l'administration bavaroise a fait relever soigneusement trois profils en travers de l'Jsar, au point où elle prévoyait l'ćlablissement du barragr de prise. Or, en ces trois points, très voisins l'un de l'autro, la section de l'cau avait une surface très sensiblement la même pour toutes les trois et égale à $12,63 \mathrm{~m}^{2}$. Le rayon moyen en était d'environ $o^{\mathrm{m}} \mathrm{i}^{2}$, et la pente superficielle du cours d'eau de 3,7 pour I noo, en sorte que le débit devait atleindre ${ }^{1}, 9 \mathrm{~m}^{3}$ par seconde, si le lil de l'Isar esl formé de cailloux roulés, ou de $16, \mathrm{I} \mathrm{m}^{3}$ si le lit peut être assimile it de la terre. Or, dans les relevés journaliers des six années d'observations, failes près de Wallgau, an pont de l'Isarhorn, et qui ont élé communiquées aux concurrents pour leurs études, le débit indiqué pour co même so seplembre 1 goo cst de $22 \mathrm{~m}^{3}$ par seconde.

Il semble donc probable que les débits en basses eaux su: lesquels les projets sont basés sont trop élevés, comme c'est du reste le cas habiuel. dans les projets dutilisation de fores molrices hydravliques. Ia realisalion de l'entreprise 
élanl chose deciché, il semble quiil n'y anail que des avantages, el point d'inconvénients, à procéder à la vérification soiguce de lexaclitule te res dóbils

Le second point, sur lequel nous désirons allirer l'attenlion, est la question de linlluence que les charriages doivent exercer sur la forme il domner anx barrages de prise. Litsar e le Risshach sont des coms d'an lorrenluenx, qui charrient des quanlités ínomes de malieres solides. Quaranle mille mètres cubes par an pour l'sear ì ce que disenl les renseignements officiels.

Les prises à exéculer doivent tre un peu différentes des prisus d'eau ordinaires. Fn effet, celleseci utilisent en lotalité les basses eaux qui sont habituollenient propres, mais en partic seulement les hantes caux, qui sont volontiers chargées de corps ćtrangers, cl cela on proporlion d'aulant plus forte que le débit est plus grand. On résout plus ou moins somplelement. Ie probleme de ne pas risquer d'ensabler les canaux de dérivation, en cherchant, dans la mesure du possible, it ne dériver en lemps de forts charriages gue les enux de surface qui sont plus claires, of en laissant les eaux do fond, soit les plus chargées, continuer leur cours dans le lit de la rivière. On purge, en outre, par le fond les caux déjà divivés. Mais, malgré les précaulions prises, l'ensablement n'est pas loujours évile, ol il n'est pas nécessame de comrir bien loin pour rencontrer des dragues disposées en permanence dans les canaux de dérivalion pour pares anx ensablements.

An Walchenser, le cas esl un pen différent. Si l'on se décidait à détourner la tolalité des eaux de l'Isar el du Rissbach pour les amener dens le lac, on pourrait résoudre avec sceurile le probleme, comme on l'a fail on Suisse en dóloumant les eaux de la kander pou les amener hans le lac de Thome. al cola déjà au XVII siècle, et au siècle suivant, eclles de In linth dans lo lac de Wallenstadt, et celle de l'Mar dans le lae de Bienne.

Il suffit de domner ì la dérivation une penle et une section crales ou ćquivalenles à ro qui existe dans la partic amont du cours d'eau, avant is point où les ensablements ont commencé à se produire, et ont obligé à faire la correction.

Pour le but poursuivi au Walchensee, il n'est pas nécessaire de prendre le tolal de toutes les crues, car cela augmenterait beaucoup la section de dérivation el constituerait une dópense excessive ol qui n'équivandrait pas an gain fait en cau accumulée. In limite du volume d'eau à détourner à lirquelle se sont arrêlés les divers auleurs de projet est assez variable. Ainsi, landis que le projet tres pondéré qui a reç le premier prix limite à 30 et $15 \mathrm{~m}^{3}$ par seconde les apports ì rehirer de l'Isar et du Rissbach, la plupart des autres élèvent

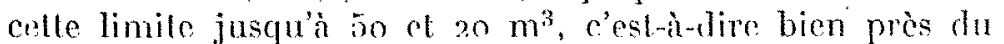
maximum observé.

La dérivalion de l'eau n'étant pas totale, il semble indiqué de l'ébablir de façon à laisser dans le lit de la rivière les caux de fond les plus chargées, of de n'envoyer dans les sonterrains que les eaux de surface.

Pour cola, il as à peu près indispensable d'avoir en travors du cours d'eau un barrage mobile, comme il en existo de nombrenx lypes, el non pas un barrage fixe, of d'installer un scuil légèrement relevé à l'entréc de la dérivation. C'cst le contraire qui a été généralement projeté, quitte ì installer une purge supplémentaire, mais nécossairemenl dérisoire après la prise.

Si lans le cas d'une disposition qui semble rationnelle, le conrs d'eau ì l'aval de la prise, ne recevant plus qu'une petite quantité d'ean propotionmellement tròs chargée, s'en- sable, le mal ne sera pas très grand et facilement réparable. Mais si c'est, comme dans la plupart des projels, le soulermin qui recoil la portion de l'cau proportionnellement la plus chargéc de corps en suspension, ou entrainés, et il s'ensable un jour de grande crue, el se remplit de gravier, lo nelloyage sera long, diffeile et coüteux. On sait, du reste, que les soutermins de dírivation d'ean qui s'ensablent, so n'est pas un mylhe, même si l'accès du gravier, sinon du sable, ost absoliment of effencement empèché.

\section{INSTALLATIONS HYDRO-ÉLECTRIQUES}

\section{USINE HYDRO-ÉLECTRIQUE DE TROLLIATTAN}

L'Etat Sucdois vient de conslruire celle usine pour distribuer l'énergic d́lectrique, dans un rayon de $150 \mathrm{~km}$, pour l'éclairage, la force motrice el même ta traction télectrique. Gefle usine ulilisern les famenses chules du Gota elf a Trollhatan, bien connues des touristes.

Le Gota elf est l'émissaire naturel du lac Venern, le plus grand d'Furope, après ceux do Russie. La superficie de ce lac est de $5568 \mathrm{~km}^{2}$, et son principal affuent est le Klar elf qui prend sa source au lac Foenumd, dans le Irodemarken. en Norvège. Le Gola ell se jette dans le Kallegal, a Golleborg, en face de l'extrómilé nord du Danemark.

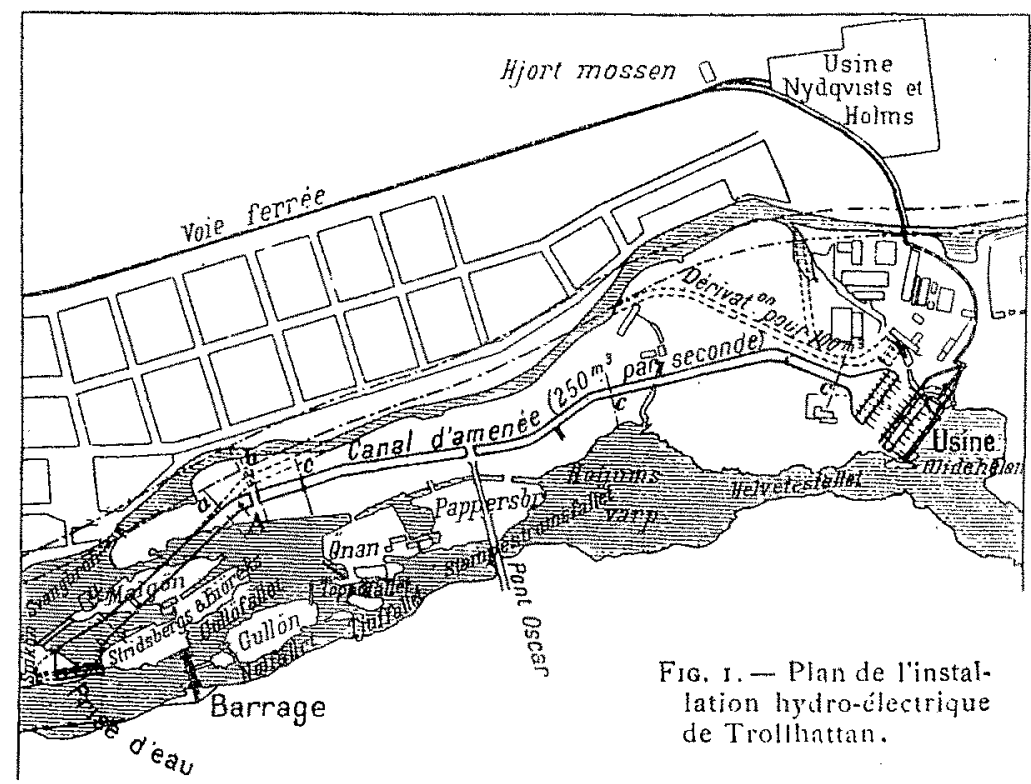

A r.́ km. à l'aval du lac Venern le Gota elf forme los chutes de Trollhallan, dont la hauleur tolale de $33 \mathrm{~m}$. se ré parlit sur une série de chules successives s'échelonnant sur une longueur de a boo $\mathrm{m}$. Ces chules sont, damont en aval.

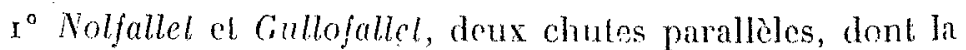
hauteur est de $7 \mathrm{~m}$, d qui sont séparces par la pelite île de Gullon

$?^{\circ}$ Toppofale et Tiujalle (chule du Voleur), deux chules ćgalement paralleles, qui ont $13 \mathrm{~m}$. de liaulcur, ct sonl sépróes par lîlol de Toppon.

$3^{0}$ Slampestromfallet, qui a $5^{n} 50$ de hanleur el se trouve an-dessous mème du pont dn roi Oscar.

$4^{\circ}$ Helvelesfallet (chute de l'Enfer), qui est haute de $7^{\text {miro }}$ th se lroure près de l'usine gónéralrice.

Un barrage établi en amonl des deux premières chutes, avec appui sur l’̣̂lo Gullon, hévivo l'eau dans un canal à ciel 